\section{Teilrevision des}

\section{Bundesgesetzes über die Krankenversicherung}

\author{
Revisionsentwurf vom 18. September 2000
}

\section{H. H. Brunner, Präsident FMH}

Zurzeit wird die Botschaft betreffend die Teilrevision des Bundesgesetzes über die Krankenversicherung vom 18. September 2000 (nachfolgend Botschaft) durch die ständerätliche SGK hinsichtlich der Beratung durch den Ständerat, den Erstrat in diesem Geschäft, vorbereitet. In diesem Zusammenhang ist auch ein in der Ärzteschaft weit verbreitetes Missverständnis auszuräumen: Als Vorlage unterliegt die Botschaft keiner Vernehmlassung, die ja im Vorfeld dieser Botschaft erfolgt ist [1]. Im Rahmen dieser Vorbereitungsarbeiten fand aber ein Hearing durch die SGK des Ständerats statt, an dem auch etliche $\mathrm{Na}$ tionalräte teilnahmen. Die FMH hatte Gelegenheit, ihre nachfolgend zusammengefasste Position zu vertreten und aufgrund der Diskussionen den Stand der politischen Diskussion und insbesondere die möglichen Entwicklungen zu analysieren. Die nachfolgenden Zeilen fassen Position und Analyse zusammen. Für ihr Verständnis wird auf den nachfolgend publizierten Text der Vorlage verwiesen.

Neben Anpassungen, die - hauptsächlich durch Rechtssprechung - zwingend geworden oder von untergeordneter Bedeutung sind, beinhaltet die Vorlage einerseits Vorschläge zur Änderung der Finanzierungsmodalitäten im KVG, die weit über den Spitalbereich hinaus wirksam wären; andererseits fasst sie Modalitäten zur Einschränkung des Kontrahierungszwanges ins Auge.

Leider hat der Bundesrat den nicht nur von der FMH immer wieder geforderten Schritt zur monistischen Finanzierung der Spitäler nicht vollzogen. Erhalten bleibt also das sogenannte duale System (Finanzierung durch die Versicherer und öffentliche Hand zu nun fix 50\%) - ein System, das klare Verantwortungen und damit auch ein wirksames (Kosten-)Management verunmöglicht. Die öffentliche Hand, vornehmlich die Kantone, verbleiben in ihrer Dreifachrolle als Spitaleigner, Spitalfinanzierer und Spitalaufsichtsbehörde in eigener Sache. Manche der für den Normalverbraucher so unverständlichen Pirouetten des schweizerischen Gesundheitswesens finden hier ihre Erklärung.

Der zumindest versuchte Schritt von der Objektzur Leistungsfinanzierung ist, nicht zuletzt im Lichte der altbekannten FMH-Forderung nach Gleichbehandlung von Spital und Arztpraxis, zu begrüssen. Verschiedene aktuelle Probleme können damit gelöst werden:
- Konfliktträchtige Begriffe wie «Allgemeine Abteilung», "Privatabteilung» etc. werden hinfällig. Entscheidend für Grundsätze und Modalitäten nach KVG ist nur, ob die Leistungen zum Leistungskatalog des KVG gehören - womit eine andere Forderung der FMH nach Inhaltsdefinition einmal mehr zu verbinden ist.

Wenig Sinn macht die Austreibung des Teufels verwirrlicher Begriffe durch den Belzebub neuer Unklarheiten. Gemeint ist die "teilstationäre Behandlung", die zu definieren auch den erlauchtesten gesundheitspolitischen Laureaten dieses Landes mit Ausnahme des Psychiatriebereichs während 15jähriger Anstrengungen nicht gelungen ist. Sinnvoll können Leistungen nur danach eingeteilt werden, ob sie ambulant (Patient schläft zu Hause) oder stationär (Patient schläft, soweit er kann, im Spital) erbracht werden; tertium non datur. Den Spitälern durch Begriffe wie teilstationär den Vorwand zu liefern, die gleiche Leistung teurer zu verkaufen, zählt zu den Lügen mit den ganz kurzen Beinen, die innerhalb kurzer Zeit durch die nun generierbaren Zahlen als solche entlarvt werden.

- Das bisher geltende Kostendeckungs- oder Kostenerstattungsprinzip führte dazu, dass die Spitaltarife nur einen Teil der anrechenbaren Kosten abgalten, dies mit von Kanton zu Kanton differierenden Modalitäten - mit der Folge, dass die gleiche Leistung von Kanton zu Kanton verschiedene Preise aufweist, die überdies noch von den Tarifen bzw. Preisen in freier Praxis abweichen. Die Einführung von TarMed lässt grüssen. Die Lösung dieses Problems durch Abgeltung der Leistung nach schweizerisch einheitlichen Tarifssystemen auch im stationären Bereich - hier mit Fallkostenpauschalen, die kurz darauf in den gesamten ambulanten Sektor Einzug halten dürften - ist deshalb zu begrüssen.

\section{Angriff des EDI auf die Zusatzversicherung}

Von vielen Seiten, nicht zuletzt auch von Ärztekreisen, wurde seit Inkrafttreten des neuen KVG moniert, der Zusatzversicherte habe für die stationäre Behandlung auch Anrecht auf anteilsmässige Zahlungen der Grundversicherung, der anzugehören ihm gesetzlich vorgeschrieben sei. Das EVG hat diese Sicht, allerdings für den Fall einer ausserkantonalen Behandlung, geschützt; obwohl entsprechende Urteile für die Behandlung im Wohnortskanton des Patienten unterblieben sind, ist die Anwendung dieses Grundsatzes für die Behandlung des Zusatzversicherten in seinem Wohnortskanton logisch zwingend. Die Botschaft verankert diesen Grundsatz nun im Gesetz (vgl. Art. 49, insbesondere Abs. 1 und 2).

Für Zusatzversicherte behandelnde Ärztinnen und Ärzte zentral ist in diesem Zusammenhang Abs. 5 dieses Art. 49; er stipuliert, dass alle Ansprüche des Spitals (und mithin auch der behandelnden Ärztinnen und Ärzte ) mit den Vergütungen nach Abs. 1-4 
des Artikels 49 abgegolten sind. Im Klartext und etwas vereinfacht: Mit dem Anteil Grundversicherung an den stationären Behandlungen wird das gesamte Leistungspaket des KVG in die Zusatzversicherung dergestalt importiert, dass eine Abrechnung dieser Leistungen über die Zusatzversicherung ausgeschlossen ist. Was der Abgeltung durch die Zusatzversicherung nach heutigen Tarifstrukturen bleibt sind Hotellerie und Nichtpflichtleistungen wie Facelifting, Operationen mit rein ästhetischer Zielsetzung an den Mammae, Haartransplantationen u.ä.

Zweifelsohne hat das Departement Dreifuss hier einen Meilenstein auf seinem Kriegszug gegen die Zusatzversicherung gesetzt, ein Kriegszug, der medial geschickt orchestriert seit Jahren systematisch geplant und ausgeführt wurde und wird. Im geschilderten Kontext dürfte es ausserordentlich schwer sein, diese Entwicklung zu verhindern, sprich die erwähnten gesetzlichen Bestimmungen zu eliminieren - um so mehr, als interessierte Teile der Ärzteschaft mit ihren Forderungen nach tarifarischer wie gesetzlicher Anbindung der Zusatzversicherung an die Grundversicherung mit behilflich waren, den Boden für diese fatale Entwicklung zu ebnen. Der Zentralvorstand hat seit mehr als zehn Jahren immer wieder vor dieser Entwicklung gewarnt, die absehbar war.

Was nun? Die Antwort auf diese Frage ist im Grunde einfach: Die Zusatzversicherung muss Leistungen abgelten, die nicht im Leistungsbereich und damit Tarifschutz des KVG liegen. Konkret:

- Basis kann nicht mehr die höhere Abgeltung von in Sozialversicherungstarifen (TarMed!) tarifierten Leistungen sein.

- Die Zusatzversicherung honoriert die persönliche ärztliche Leistung der Ärztinnen und Ärzte, die vom zusatzversicherten Patienten nach dem Prinzip der freien Arztwahl für die Behandlung ausgewählt werden.

- Das Leistungsspektrum der sozialen Grundversicherung muss reduziert werden mit der Zielsetzung, die soziale Grundversicherung schwergewichtig zur Finanzierung kostenträchtiger Behandlungen einzusetzen (eine Idee, die wahrlich nicht neu ist und nur nun endlich einmal in die Tat umgesetzt werden müsste).

Entsprechende Zusatzversicherungsmodelle wurden bereits konzipiert und sind durchaus auf das Interesse der Zusatzversicherer gestossen, deren Überlegungen in ähnlicher Richtung gehen. Entscheidend ist, dass die Arbeiten nicht mehr in fixierter Perspektive auf das Unwesentliche sorgsam gehütete Prärogative einzelner Ärztegruppierungen sein können, sondern aus einem Konsens der gesamten Ärzteschaft gemeinsam geleistet werden müssen.

\section{Zur Einschränkung des Kontrahierungszwangs}

Die in der Vernehmlassung vorgeschlagene Einschränkung des Kontrahierungszwanges hat - wen wundert's - nur gerade die Zustimmung der Kran- kenversicherer gefunden. Gemäss Botschaft soll mit identischem Text diese Einschränkung des Kontrahierungszwanges nun auf die über 65jährigen Leistungserbringer beschränkt werden. Die im Begleittext angedeutete Begründung, mit dieser Regelung einen Beitrag zur Qualitätssicherung zu leisten, ist dermassen dürftig, dass sie in geradezu beleidigender Weise nicht einmal ernsthaft die Anstrengung unternimmt, die wahre Motivation zu verbergen: Auf dem Buckel der Alten, von denen man annimmt sie würden sich nicht wehren und auch nicht über eine Lobby verfügen, wird das Prinzip des aufgehobenen Kontrahierungszwanges nach dem Diktat der Krankenversicherer so verankert, dass es kurz darauf nach dem Gebot der Gleichbehandlung auf alle Ärztinnen und Ärzte ausgedehnt werden kann - auch diejenigen jungen Kolleginnen und Kollegen, die sich ob der aus dem System gekippten älteren Kolleginnen und Kollegen bessere Zukunftsaussichten versprechen mögen. Eine Hoffnung, die sich in sehr kurzfristigem und zudem überschätztem Gewinn erschöpfen würde.

Im einzelnen ist folgendes festzuhalten:

- Die Regelung würde ca. 800-900 Ärztinnen und Ärzte betreffen, die durchschnittlich noch ein Pensum von ca. 30\% (bezogen auf TarMed-Normen) arbeiten. Diese Ärztinnen und Ärzte erbringen praktisch nur noch Leistungen, die tarifarisch den sogenannten Basisgrundleistungen zuzuordnen sind. Operative oder interventionelle Tätigkeiten stellen eine seltene, dann aber meist sinnvolle, Ausnahme dar.

- Es existieren keine Daten, die bei Leistungserbringern gemäss KVG den Zusammenbruch der geistigen und manuellen Fähigkeiten an deren 65. Geburtstag dokumentieren würden. Die Einsicht, dass solche Stufen nicht existieren, ist einer der massgeblichen Gründe für die allerorten in Verwirklichung stehende "retraite à la carte», die nun gerade für "medizinische Leistungserbringer» ausgeschlossen sein soll. Pikantes Detail: Im Rahmen von Notfallbehandlungen, die ausserhalb des eingeschränkten Kontrahierungszwanges stehen, scheinen nach Botschaft die in Demenz Dahindämmernden schlagartig ihre gesamten Fähigkeiten zurückzuerlangen ...

- Die Patientinnen und Patienten werden nicht gezwungen, sich durch ältere Ärztinnen und Ärzte behandeln zu lassen. Die Elimination dieser Ärztinnen und Ärzte würde aus der Sicht ihrer Patienten gut funktionierende Patienten-Arzt-Beziehungen zerstören und stehen so betrachtet auch in eklatantem Widerspruch zum von den Gesetzesredaktoren so lauthals vertretenen Wettbewerbsmodell.

- Die Ärztinnen und Ärzte fühlten sich immer den sogenannten liberalen Berufen zugehörig, ein Begriff, der u.a. professionelle Selbstbestimmung meint. $\mathrm{Zu}$ den Essentialia dieser Berufe gehört, dass ihre Angehörigen den Rückzug aus der beruflichen Tätigkeit inhaltlich wie zeitlich selber festlegen. Die Vorlage würde diesen Zeitpunkt de facto gesetzlich festlegen und damit einen Schritt 
mehr in Richtung einer Verbeamtung der Ärzteschaft bedeuten. Weite Kreise befürworten heute aus nicht immer edlen Motiven eine solche Verbeamtung; möglicherweise haben sie sich $\mathrm{zu}$ wenig die Frage gestellt, ob sie sich den zwangsläufig aus dieser Entwicklung hervorgehenden Ärztefunktionär an ihrem eigenen Krankenbett wünschen würden.

Seitens der Ärzteschaft bzw. der FMH wurde immer gefordert, dass Kriterien für den Ausschluss von Ärztinnen und Ärzten aus Versicherungsverträgen klar festgelegt werden sollten. Solche klar definierten Kriterien und ihre konsequente Handhabung werden von der FMH nicht zuletzt deshalb gewünscht, weil damit eine Einschränkung des Kontrahierungszwanges vermieden werden könnte. Solche Ausschlusskriterien werden nun in Art. 59 des Botschaft formuliert; sie werfen aber mehr Fragen auf als sie solche beantworten:

- Was heisst Überarztung? Wer legt die genauen Kriterien fest? Welches sind insbesondere die Daten, die den Entscheiden zugrunde liegen? Nach den leidvollen Erfahrungen mit Rückforderungsverfahren akzeptiert die FMH hier keine Formulierungen ins Ungefähre.

- Nichtbeachtung des Tarifschutzes ist ein problematischer Ausschlussgrund, solange die Konturen dieses Tarifschutzes noch unbestimmt bzw. verschiedene gerichtliche Entscheide offen sind.

- Das Gebot der Informationspflicht belässt den Arzt im Zwiespalt zwischen der Skylla des Datenschutzes und der Charybdis der Versichererforderungen, ohne dass das Gesetz die List des Odysseus bergen würde, die ihn aus dem Zwiespalt führen könnte.

- Und dann einmal mehr: die Vergünstigungen. Wiederum würde es der Gesetzgeber unterlassen, diesem Fliegenden Holländer Gestalt und Farbe zu geben, mit der Folge, dass die Bestimmung nicht umsetzbar wäre. Was wiederum den Ärztinnen und Ärzte zum medial so wirksamen Vorwurf gemacht werden kann. Und was wiederum vielleicht sogar der Zweck des Ganzen ist.

Ohne Präzisierung lehnt die FMH diese Bestimmungen ab: Sie sind zwangsläufig wirkungslos, bringen aber die Ärzteschaft in die kafkaeske Situation des ohne Rechtfertigungsmöglichkeit ewig Schuldigen.

Zum jetzigen Zeitpunkt ist das Schicksal dieser Vorlage offen: Sie stösst auf massive Ablehnung der Kantone, die, nicht zu Unrecht, eine Minderung ihrer Prärogativen und massive zusätzliche Belastungen durch den Vollzug befürchten. Die Opposition vieler vornehmlich bürgerlicher Parlamentarier ist nicht geringer, so dass der Vorlage das Schicksal einer vollständigen oder - de facto - teilweisen Rückweisung droht.
Sollten mit einer Rückweisung der Ärzteschaft unliebsame Dispositionen dahinfallen, so wäre dies bestenfalls ein fraglicher Gewinn auf Zeit und sicher kein Grund, entspannt zurückzulehnen: Die grosse Mehrheit der eidgenössischen Parlamentarier empfindet die aktuelle verfahrene Situation des schweizerischen Gesundheitswesens als zunehmend untragbares Malaise, das einer umfassenden und durchgreifenden Therapie durch den Bund bedarf. Diese Therapie wird gerade für die Ärzteschaft einschneidend sein.

Die Vorlage rühmt sich, einen wesentlichen Beitrag zu einem wettbewerblichen Modell des Gesundheitswesens darzustellen. Einmal bleibt aber fraglich, ob dieses Wettbewerbsmodell überhaupt zu einem sinnvollen Kostenmanagement beitragen würde. Die positive Antwort auf diese Frage dürfte schwer fallen, weil bis jetzt in keinem Land diese Hypothese stützende Evidenz gewonnen wurde. Die Frage zielt aber noch viel tiefer: Ist es aus grundsätzlich staatspolitischen und ethischen Überlegungen überhaupt zulässig, das Gesundheitswesen nach einem Wettbewerbsmodell zu organisieren? Sollen ärztliche Leistungen auf einem wie auch immer strukturierten Markt angeboten werden? Wird so nicht die «Numismatik», die man gewissen Ärztinnen und Ärzte zu Recht vorwirft, zum alles dominierenden Grundprinzip erhoben? Es scheint, dass sich immer mehr Parlamentarier - erfreulicherweise - diese Fragen stellen und eine Neuorientierung der schweizerischen Gesundheitspolitik verlangen. Wir Ärztinnen und Ärzte sind aufgerufen, hier mitzuwirken. Die erwähnte Therapie könnte dann auch etwas in unserem Sinne konzipiert werden ...

\section{Literatur}

1 Brunner HH. Teilrevision des Bundesgesetzes über die Krankenversicherung, ergänzendes Vernehmlassungsverfahren. Antwort der FMH zu Handen der Vorsteherin des Eidgenössischen Departementes des Innern EDI, Frau Bundesrätin Ruth Dreifuss. Schweiz Ärztezeitung 2000;81(34):1829-37.
} 